\title{
EVALUATION DU POTENTIEL PRODUCTIF DES EXPLOITATIONS AGRICOLES DANS LES MONTS DE TLEMCEN (NORD-OUEST DE L'ALGERIE)
}

\author{
M. Hattab, A. Gaouar \\ Université de Tlemcen. Faculté des Sciences de la Nature et de la Vie et Sciences de la \\ Terre et de l'Univers. Département d'Agronomie. Rocade 2, BP N 119. Tlemcen \\ (Algérie). \\ htb83-agromourad@hotmail.fr
}

(Received August 2016 - Accepted September 2018)

\section{RESUME}

M. Hattab, A. Gaouar. 2019. Evaluation du potentiel productif des exploitations agricoles dans les monts de Tlemcen (nord-ouest de l'Algérie). Journal Scientifique Libanais. 20(1): 71-88.

La population montagnarde algérienne reste tributaire surtout d'une agriculture fragile. À cet effet, le présent travail consiste à effectuer une évaluation approfondie de la situation actuelle des exploitations agricoles, qui sont fortement fragilisées par différentes contraintes dans les monts de Tlemcen, afin d'évaluer leur potentiel productif. Les résultats obtenus ont montré que les exploitations enquêtées quoiqu'elles appartiennent à un même ensemble montagneux, elles se distinguent par des particularités différentes d'une région à une autre. Certaines exploitations sont orientées vers le model d'exploitation familiale à cause de leur morcellement répété et du manque de main d'œuvre salariée. D'autres exploitations sont obligées de développer une stratégie de survie basée essentiellement sur une céréaliculture extensive combinée surtout avec l'élevage ovin. Dans l'ensemble, les moyens de production actuels de toutes les exploitations enquêtées sont insuffisants pour qu'elles puissent résister au climat irrégulier, à la configuration difficile des terrains et aux fluctuations du marché.

Mots-clés : monts de Tlemcen, potentiel productif, exploitations enquêtées, contraintes.

http://dx.doi.org/10.22453/LSJ-020.1.071-088 


\begin{abstract}
M. Hattab, A. Gaouar. 2019. Evaluation of the productive potential of farms in Tlemcen Mountains (north-western Algeria). Lebanese Science Journal. 20(1): 7188.
\end{abstract}

The Algerian mountain population still depends primarily on a fragile agriculture. To this end, the present work is to carry out a thorough evaluation of the current situation of farms, which are strongly weakened by various constraints in the mountains of Tlemcen, in order to evaluate their productive potential. The results showed that the surveyed farms although they belong to the same mountain range, they are distinguished by different features from one region to another. Some farms are oriented to the family farm model because of their repeated fragmentation and the lack of hired labor. Other farms are forced to develop a survival strategy based mainly on extensive cereal combined especially with sheep farming. Overall, the current means of production of all surveyed farms are insufficient for them to resist irregular climate, the difficult configuration of the land and to market fluctuations.

Keywords: mountains of Tlemcen, productive potential, surveyed farms, constraints.

\title{
INTRODUCTION
}

Les montagnes recouvrent pratiquement le quart de la surface terrestre du globe et abritent environ $12 \%$ de la population mondiale (FAO, 2013). Elles représentent des centres uniques de diversité biologique et culturelle. Elles fournissent aussi des ressources vitales aux zones qu'elles occupent et même pour les plaines (Castelein et al., 2006). Cependant, les populations montagnardes sont parmi les plus pauvres et les plus mal nourries de la planète, et les régions montagneuses parmi les plus exposées à la dégradation de l'environnement (FAO et CIHEAM, 2007).

À l'instar de beaucoup de pays dans le monde renfermant de massifs montagneux, l'Algérie quant à elle comprend deux grands ensembles de montagnes : les chaînes de l'Atlas au Nord et celles du Sahara au Sud. En effet, afin d'assurer la protection et le développement de ces zones montagneuses, l'Etat algérien auparavant a fait simplement usage des lois existantes, notamment celles régissant les ressources naturelles (forêt, eaux, sols, etc.) qui bien que partiellement applicables à ces régions défavorisées, avaient un champ spatial bien plus vaste. Ce n'est que récemment que ces zones, à contraintes spécifiques, ont retenu l'attention du législateur, avec l'adoption de la loi 04-03 du 23 juin 2004 relative à la protection des zones de montagne. Celle-ci offre un cadre juridique général pour la sauvegarde et l'aménagement des régions montagneuses dans une optique de développement durable. 
En 2006, l'Etat algérien a adopté une nouvelle politique de développement rural qui a venu consolider la loi 04-03 en ciblant prioritairement les zones où les conditions de production sont les plus difficiles pour les agriculteurs (zones de montagne, zones steppiques, oasis de Sahara). Avec l'avènement de cette Politique de Renouveau Rural (PRR), l'intervention publique en milieu rural en général et en zones de montagne en particulier se conçoit différemment. Après avoir été descendante et sectorielle, elle est devenue transversale, de proximité, ascendante, intégrée, participative et décentralisée (MADR, 2006).

Après 10 ans d'expérience, il se trouve nécessaire de voire l'impact des projets de développement rural ayant été réalisés jusqu'à présent, notamment en ce qui concerne les activités agricoles qui sont considérées les principales sources de revenu pour les populations montagnardes. Pour cela, l'objectif de ce travail consiste à évaluer le potentiel productif actuel des exploitations agricoles dans quelques zones montagneuses (Aïn Fezza, Sidi Djilali et El Gor) appartenant aux monts de Tlemcen. Cette évaluation nous permettra par la suite d'identifier les acquis à promouvoir ainsi que les insuffisances à rattraper tout en respectant les potentialités et les contraintes caractérisant ces zones de montagne.

\section{MATÉRIELS ET MÉTHODE}

Le recours à des enquêtes auprès des agriculteurs est une action primordiale dans toute approche visant à étudier un milieu donné du monde rural. Pour cela, la méthode de travail qu'on a adoptée consiste à mener des enquêtes auprès des exploitations agricoles dans trois régions montagneuses (Aïn Fezza, El Gor et Sidi Djilali) appartenant aux monts de Tlemcen qui se situent au nord-ouest de l'Algérie. La première zone d'Aïn Fezza se situe dans l'exposition nord des monts de Tlemcen, et les deux autres zones d'El Gor et de Sidi Djilali se situent dans l'exposition sud de cet ensemble montagneux (Fig. 1).

L'outil de ces enquêtes est un questionnaire formulé et conçu d'une manière à ce que l'interviewé puisse se prononcer sur l'état actuel du potentiel productif de son exploitation agricole. Etant donné que l'objectif qu'on veut atteindre par cette enquête est un objectif bien déterminé, on a choisi pour cela un questionnaire fermé afin de recueillir les réponses préalablement visées.

Ainsi, le questionnaire a concerné premièrement la nature juridique ainsi que la taille des exploitations enquêtées pour voir leur impact sur le fonctionnement de ces exploitations; deuxièmement, les systèmes de production pratiqués que ce soient végétale ou animale; et finalement, les moyens de production existants (sources 
d'irrigation, matériels agricoles, les infrastructures agricoles, les intrants agricoles et la disponibilité de la main d'œuvre salariée).

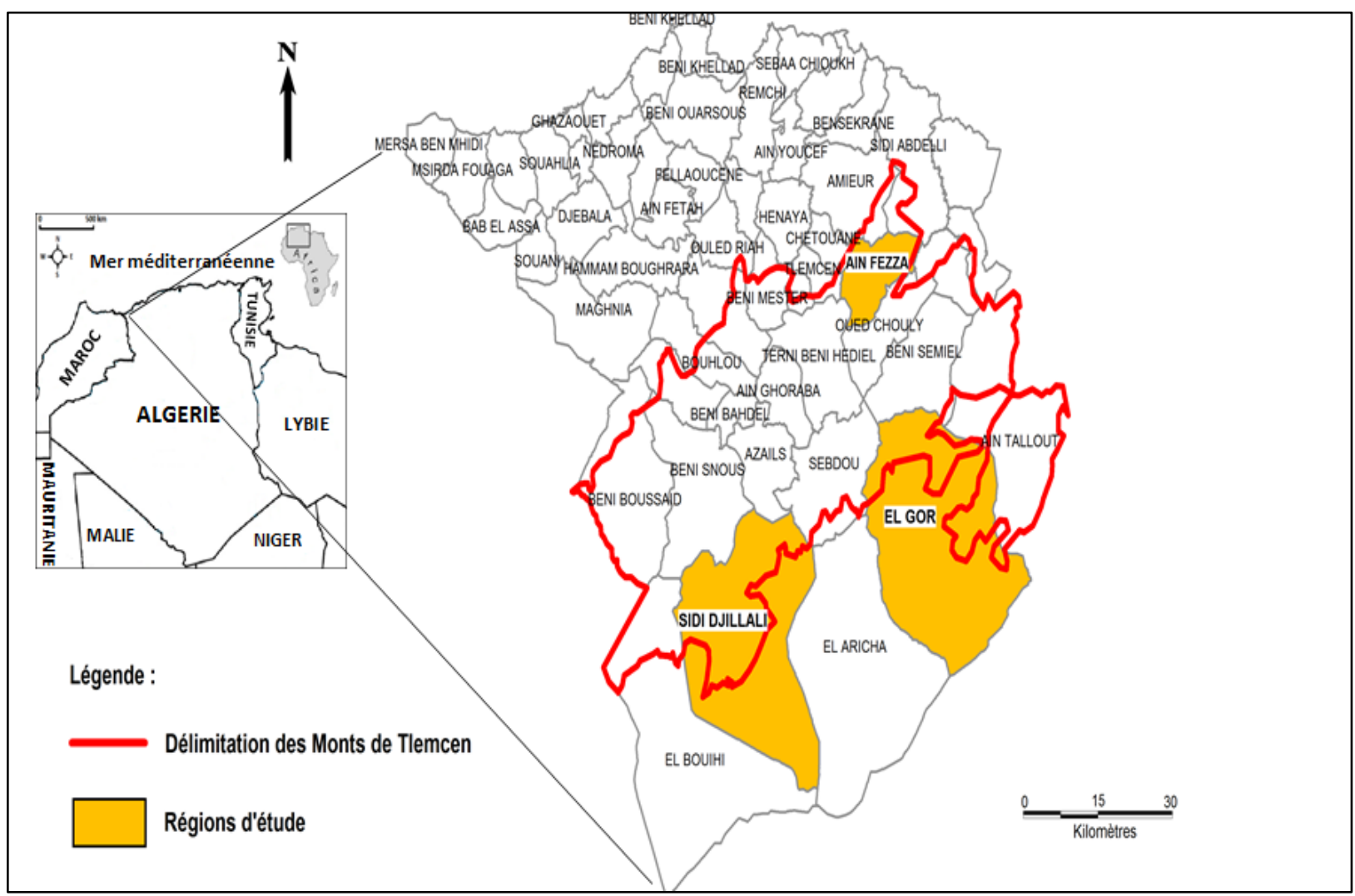

Figure 1. Localisation des trois régions d'étude dans les monts de Tlemcen.

L'échantillon enquêté a regroupé 122 exploitations agricoles réparties selon les trois régions étudiées de la façon suivante : Aïn Fezza 34 exploitations ; El Gor 53 exploitations ; Sidi Djilali 35 exploitations.

Ces exploitations enquêtées ont été choisies d'une façon à couvrir autant que possible le territoire de la région à étudier afin de refléter plus ou moins les structures foncières et les systèmes de production les plus rencontrés dans cette région. On a veillé aussi que tous les types de la nature juridique des exploitations soient pris en considération; donc, les exploitations de nature juridique différente et gérée par un même exploitant seront prises séparément. De même, la personne à interroger était le 
chef de l'exploitation, donc c'est celui qui s'occupe de la gestion et surtout de la prise de décision quant à l'investissement.

\section{RÉSULTATS ET DISCUSSION}

\section{Nature juridique des exploitations agricoles enquêtées}

La clarification de la nature juridique des exploitations agricoles en Algérie est une opération fondamentale au bon fonctionnement des projets de développement agricole. À Aïn Fezza, où la majorité des exploitations enquêtées sont des terres privées (Fig. 2), elles sont dotées, par conséquent, d'un titre de propriété leur permettant l'obtention de subventions et de crédits bancaires alloués par l'État. Par contre, à El Gor et à Sidi Djilali, la majorité des exploitations agricoles sont des terres collectives type "arch", c'est-à-dire des terres des tribus dont la nature juridique, selon Benmoussa (2013), n'est pas claire jusqu'à présent, elles sont dépourvues d'un acte de propriété, ce qui entrave énormément la participation de l'État à leur financement.

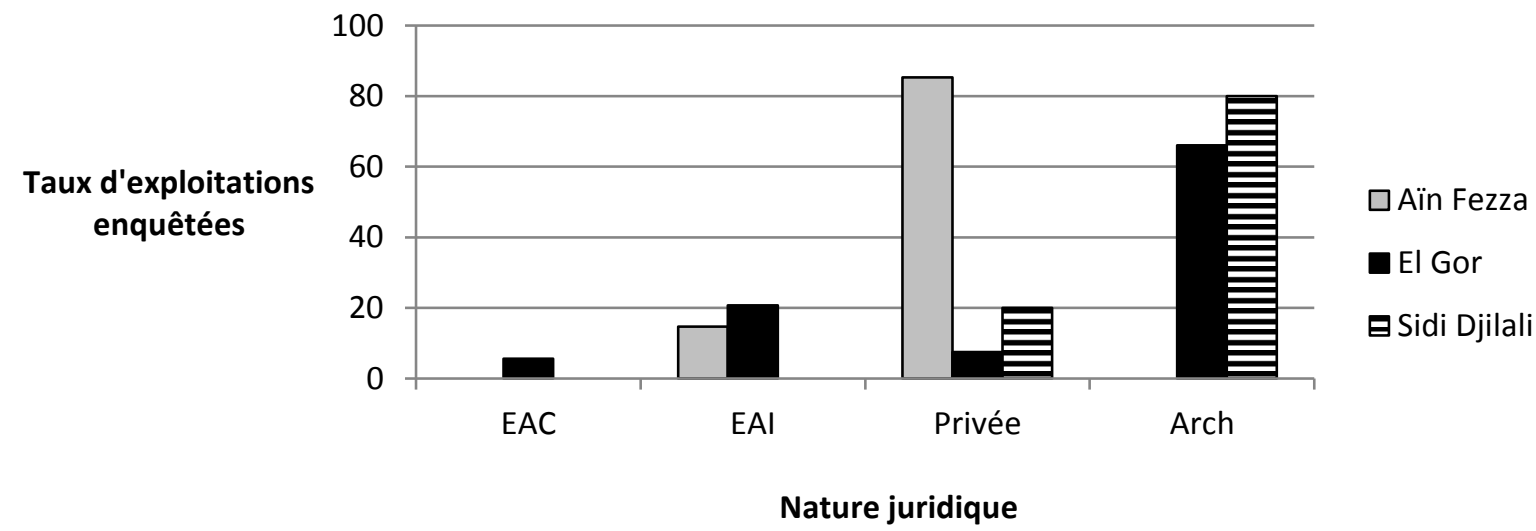

Figure 2. Nature juridique des exploitations agricoles enquêtées par régions d'étude.

EAC: Exploitation agricole collective appartenant au domaine privé de l'Etat. EAI : Exploitation agricole individuelle appartenant au domaine privé de l'Etat. Arch : Terres collectives tribales.

\section{La taille des exploitations agricoles enquêtées}

Du fait qu'elles sont des terres privées aptes à être partagées au profit des héritiers de leurs propriétaires, un bon nombre d'exploitations agricoles à Aïn Fezza se sont trouvées, de génération à génération, dans un état de morcellement très accentué. 
C'est ce qu'on a enregistré après l'examen de la taille des superficies des exploitations enquêtées où celles ayant une superficie inférieure à 05 ha (voire moins de 01 ha dans beaucoup de cas) dominent par rapport aux autres tailles de superficie (Fig. 3). Cette situation peut limiter la productivité de la terre et du travail par rapport à celle des cultures mécanisées sur des surfaces étendues (Jouve, 2001 ; Niroula et Thapa, 2005 ; Todorova et Lulcheva, 2005 ; Latruffe et Piet, 2014). En outre, ces exploitations morcelées qualifiées d'exploitations familiales constituent, d'après Sahli (2001), un handicap à l'aménagement du territoire dans ces zones montagneuses. À El Gor et à Sidi Djilali, au contraire, la majorité des exploitations ont des tailles de moyenne à grande superficie allant de 10 ha à plus de 100 ha.

Il est à signaler aussi, qu'au niveau de ces trois régions, les terres des exploitations enquêtées se localisant, en grande partie, aux piedmonts et aux vallées se caractérisent par une inclinaison modérée facilitant la mécanisation pour celles ayant une superficie suffisante. Les terres accidentées sont rarement exploitées, et pour les mettre en valeur elles nécessitent des moyens couteux hors des capacités limitées des petits agriculteurs montagnards.

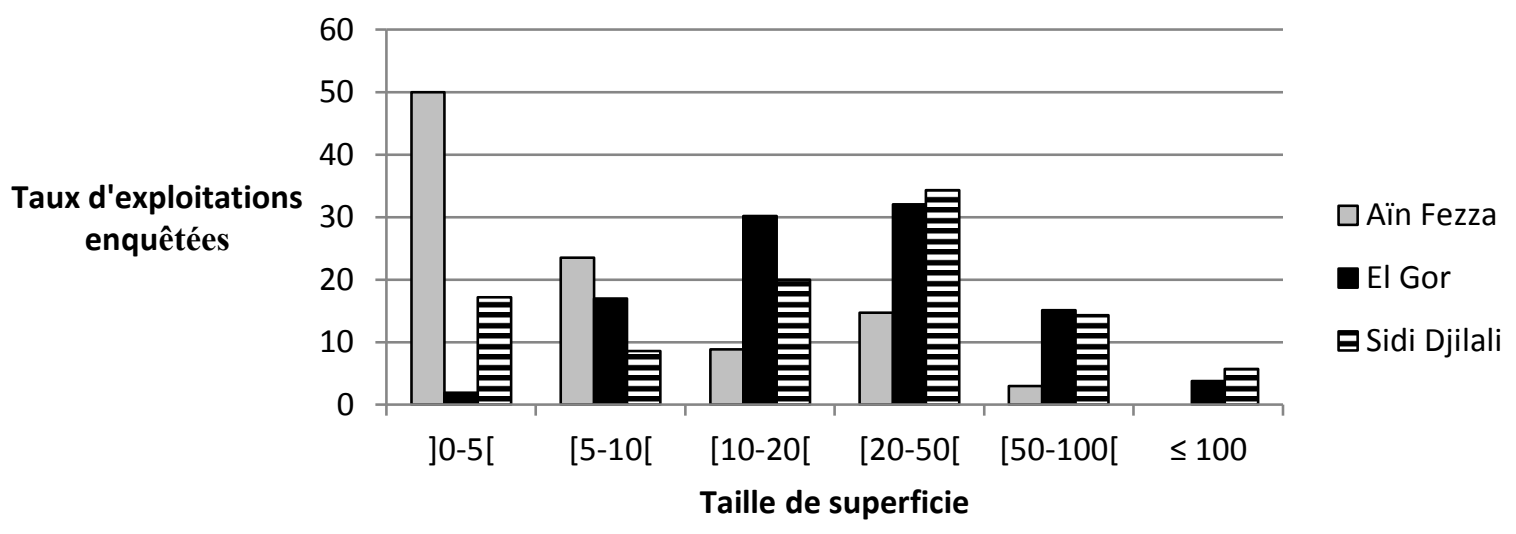

Figure 3. Taille des exploitations agricoles enquêtées par régions d'étude.

\section{Les systèmes de production}

La Figure 4 met en évidence une nette différence entre la région d'Aïn Fezza d'une part et les régions d'El Gor et de Sidi Djilali d'autre part. En fait, à Aïn Fezza, à part la viticulture, toutes les autres cultures (céréales, fourrages, légumes secs, maraîchage, arbres fruitiers) trouvent une place dans cette région mais avec des proportions différentes. Cette situation est favorisée par la richesse hydrique en cette zone. En effet, en plus de la tranche d'eau pluviale assez notable qui se précipite annuellement en cette région, la nature géomorphologique du terrain renferme 
également des résurgences et des quantités remarquables d'eaux souterraines assez faciles à être mobilisées par des forages notamment (Fig. 5), c'est ce qui a donné aux agriculteurs de cette région un choix multiple quant à l'utilisation de leurs terres notamment en cultures exigeantes en eau telles que les cultures maraîchères et les arbres fruitiers. L'irrigation permet, selon Huang et al. (2006); Angeliaume (2011) et Mihailović et al. (2014), de diversifier les cultures, améliorer la productivité des exploitations et faciliter la stabilisation des productions. Elle permet aussi d'atténuer les aléas des variations climatiques (FAO, 2004 ; Selmi et al., 2005).

Les systèmes de production pratiqués par les exploitations agricoles à El Gor et à Sidi Djilali se caractérisent par une dominance des céréales et des fourrages conduits à l'extensif (Fig. 4). La faible quantité des précipitations annuelles jointe à presque une absence de forages ou de sources naturelles (Fig. 5) ont accentué le déficit hydrique en ces deux régions montagneuses à climat aride, ce qui a obligé les agriculteurs de se limiter forcément à une agriculture très extensive représentée notamment par des céréales et des fourrages peu productifs dont le rendement moyen ne dépasse rarement les 15 quintaux/ha, et/ou par quelques petits vergers d'arboriculture rustique d'olivier et d'amandier peu exigeants en eau.

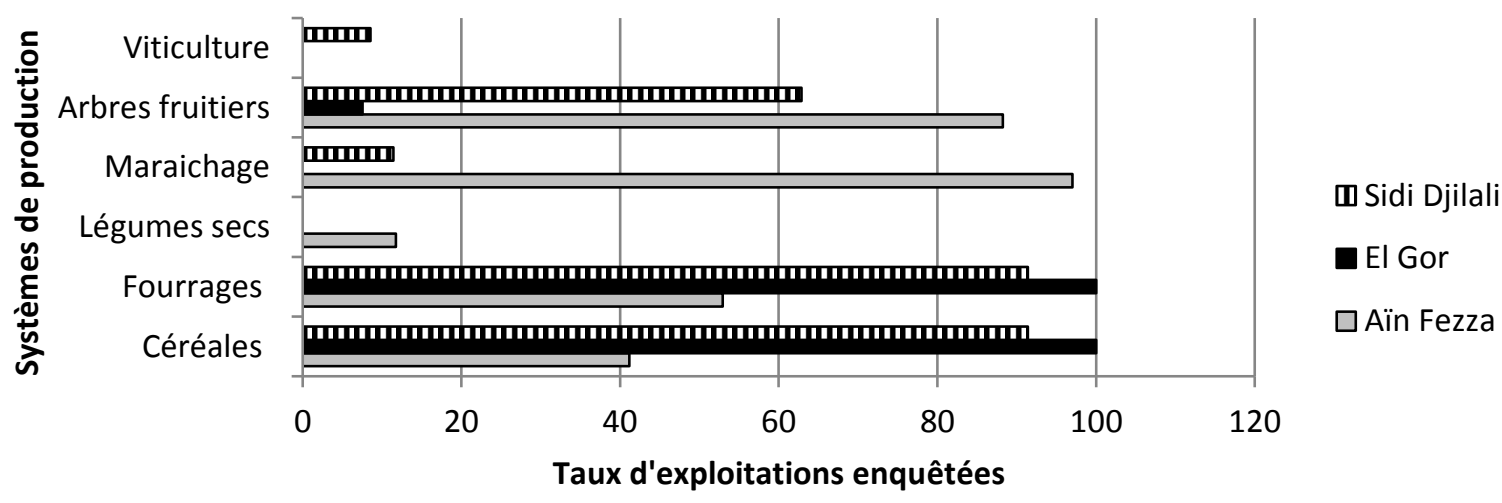

Figure 4. Systèmes de production pratiqués par les exploitations agricoles enquêtées. 


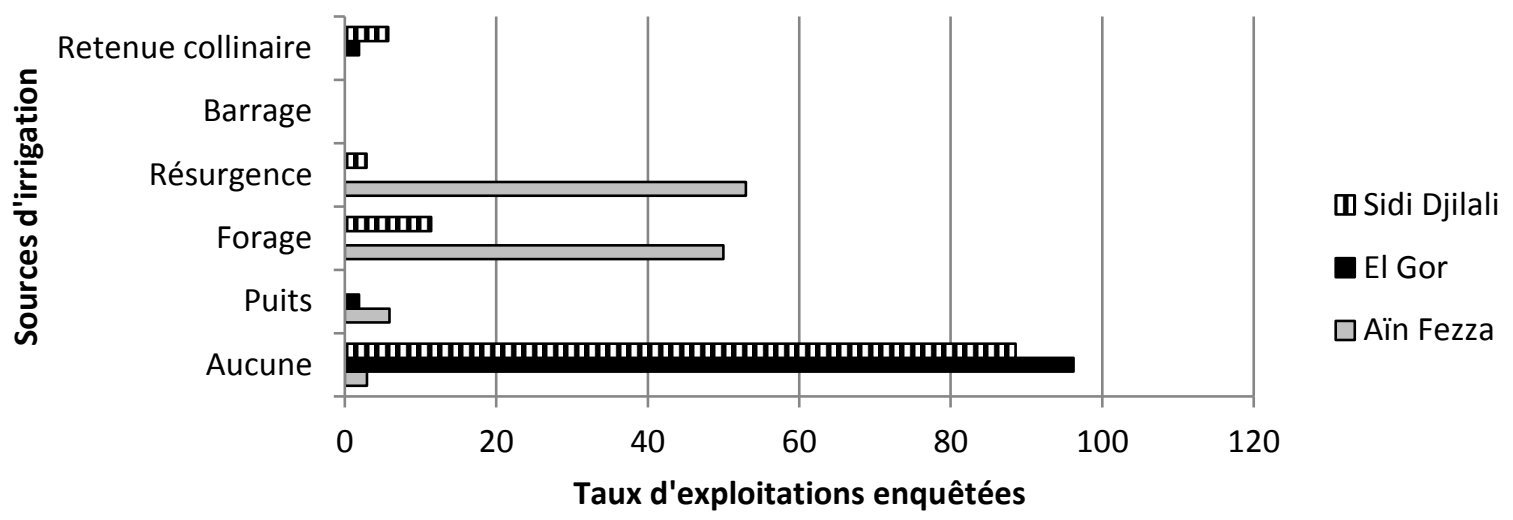

Figure 5. Taux d'exploitations agricoles enquêtées ayant de sources d'irrigation.

\section{La pratique de l'élevage ovin, bovin, caprin, avicole et apicole}

Il est tout à fait clair que la pratique de l'élevage, surtout ovin, est plus développée dans les régions d'El Gor et de Sidi Djilali par rapport à celle dans la région d'Aïn Fezza qui reste à titre familial (Fig. 6 et 7). Le caractère pastoral des régions steppiques d'El Gor et de Sidi Djilali a permis à certaines exploitations agricoles de combiner leur système de production, basé essentiellement sur une céréaliculture extensive, avec l'activité de l'élevage à grande échelle.

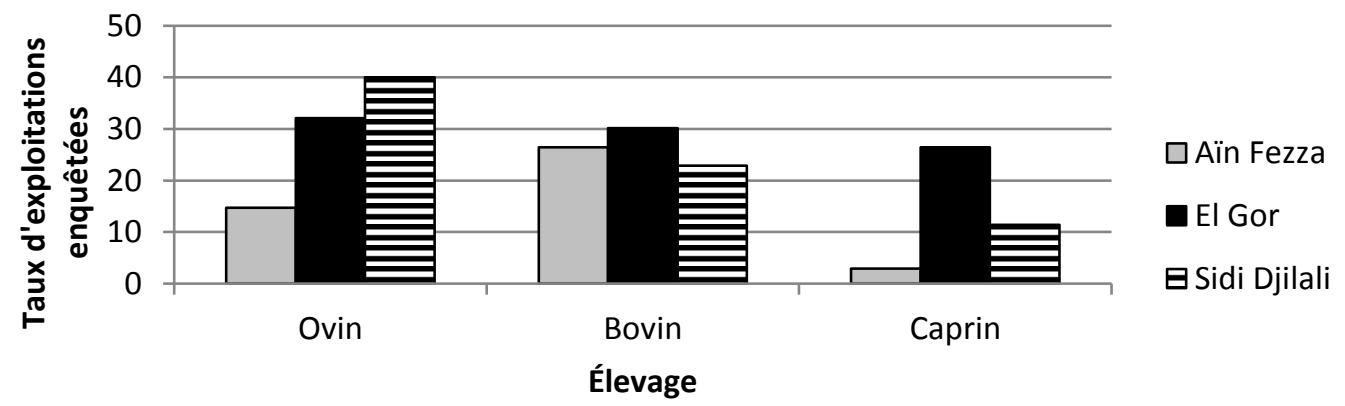

Figure 6. Taux d'exploitations agricoles enquêtées pratiquant l'élevage. 


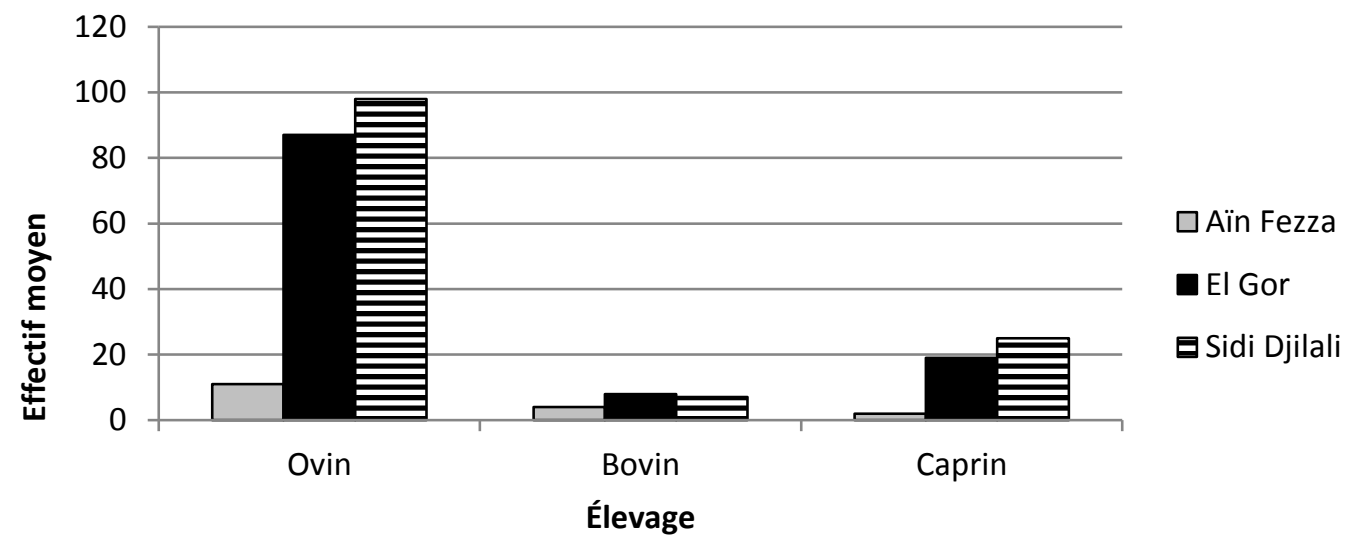

Figure 7. Effectif moyen de l'élevage ovin, bovin et caprin.

En ce qui concerne l'aviculture et l'apiculture, elles sont très peu développées dans les trois régions d'étude (Fig. 8). Il est à signaler que l'aviculture se pratique le plus souvent d'une manière traditionnelle en petit élevage. L'élevage de toute sorte confondue peut engendrer une source de revenu complémentaire très appréciable pour les petits agriculteurs dans ces régions montagneuses. Cette combinaison de l'élevage avec l'agriculture représente une sorte de diversification des productions agricoles qui est fortement recommandée en ces zones défavorisées pour pallier aux rendements faibles des cultures tributaires d'un climat aride à précipitations faibles et irrégulières. La diversification des productions agricoles est une bonne solution pour maintenir l'agriculture fragile en zones de montagne et pour qu'elle puisse s'adapter aux aléas climatiques et aux risques des fluctuations du marché (Simon, 1997 ; Hammami et Bechir Sai, 2002 ; Revel et al., 2002 ; Simon, 2002 ; Barnaud et al., 2006 ; Lin, 2011). 


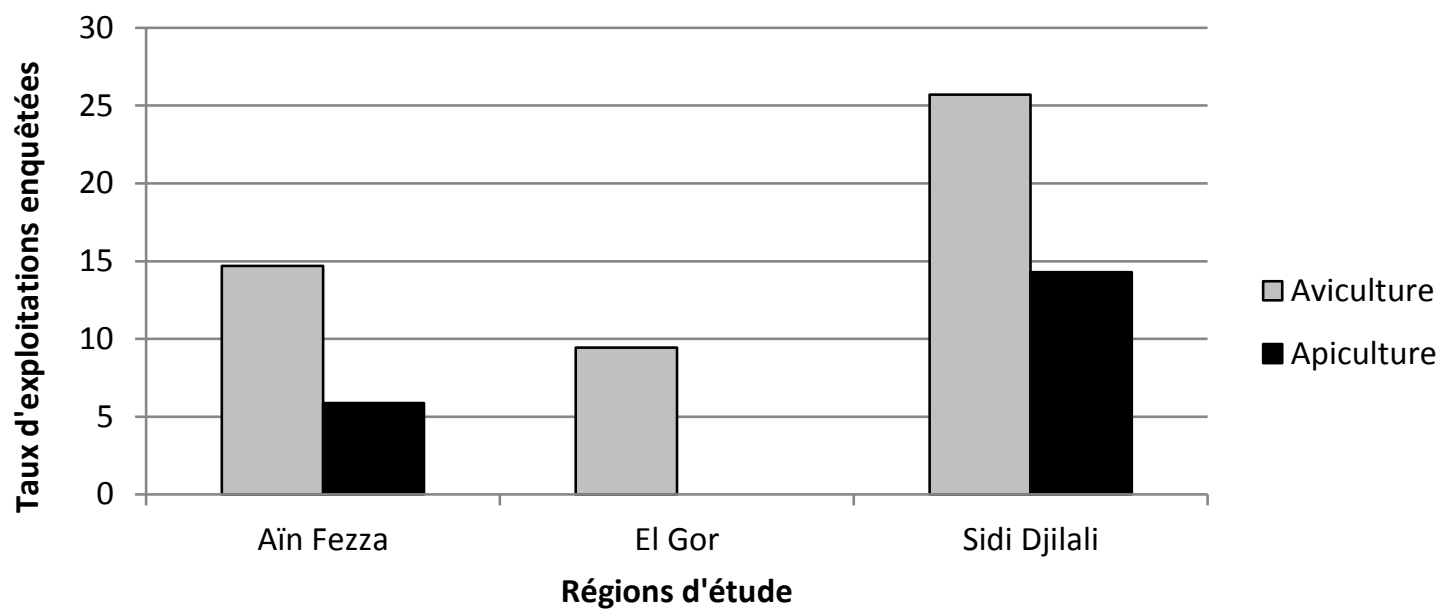

\section{Figure 8. Taux d'exploitations agricoles enquêtées pratiquant l'aviculture et l'apiculture.}

L'insuffisance ou l'absence de certains types de matériels ont obligé les exploitations agricoles de faire le recours à leur location, notamment les matériels de base nécessaires à la traction, à la préparation du sol, à la récolte et au transport, qui sont des actions constituant, pour les agriculteurs, la limite inférieure à la réussite d'un système de production donnée. Certains autres matériels sont pratiquement inexistants, soit à cause des capacités limitées de ces petits agriculteurs montagnards qui ne leur permettent pas l'achat ou la location des matériels de semis, de traitement, de fertilisation et d'amendement, soit à cause des handicaps naturels comme les cas des tailles réduites des exploitations agricoles à Aïn Fezza et des terrains à relief accidenté entravant l'accès des engins qui sont substitués souvent par un travail manuel et/ou par la traction animale (Fig. 10). Lhoste et al. (2010) a attiré l'attention sur la place de la traction animale, bien que très ancienne, qu'elle n'est pas démodée et restée d'actualité dans de nombreux pays. Les animaux contribuent encore significativement à réduire la pénibilité des travaux agricoles et de diverses autres activités, comme les transports. Ils permettent ainsi d'améliorer les conditions de travail et les revenus dans les petites exploitations agricoles (Lawrence et al., 1997 ; Belal et al., 2015). 


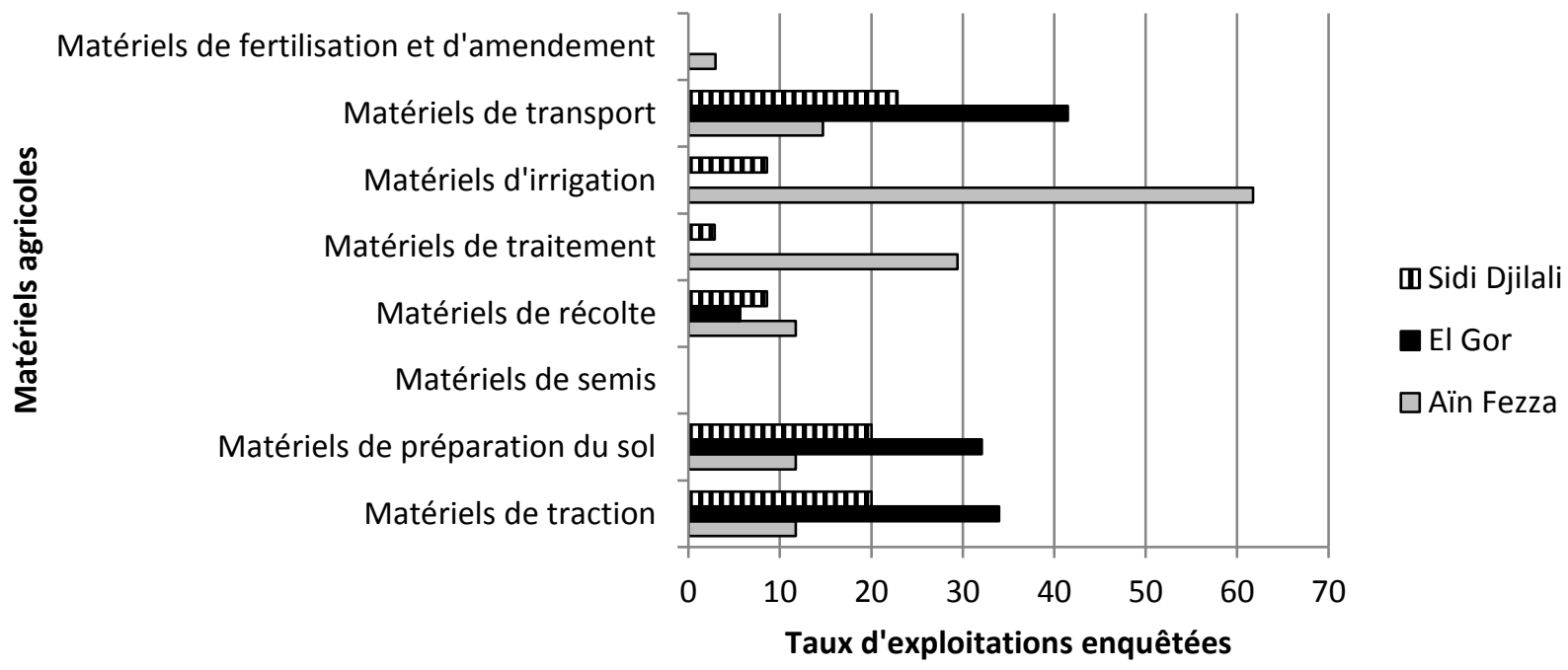

Figure 9. Taux d'exploitations agricoles enquêtées dotant de matériels agricoles.

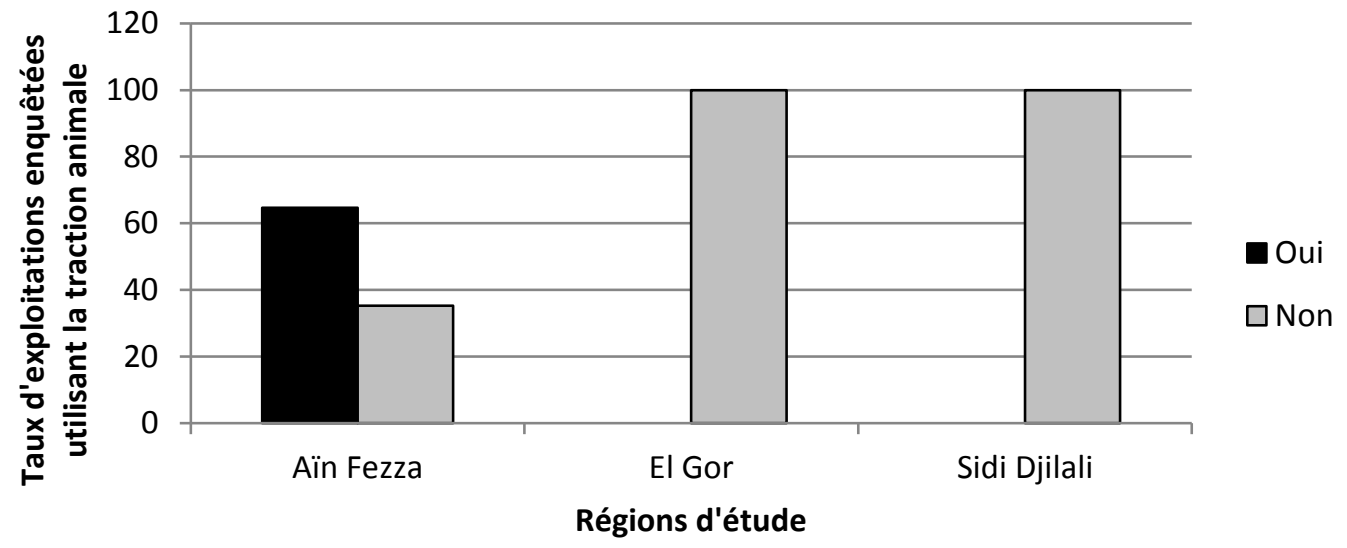

Figure 10. Taux d'exploitations agricoles enquêtées utilisant la traction animale.

\section{Les infrastructures agricoles}

Dans l'ensemble, les exploitations agricoles dans les trois régions d'étude sont moins dotées en infrastructures agricoles, et celles qui sont existées se limitent seulement à quelques bergeries et étables (Fig. 11). Le déficit en infrastructures a obligé beaucoup d'agro éleveurs, en particulier dans les régions d'El Gor et de Sidi Djilali, de loger leur cheptel sous abris simples entourés de clôtures métalliques. 


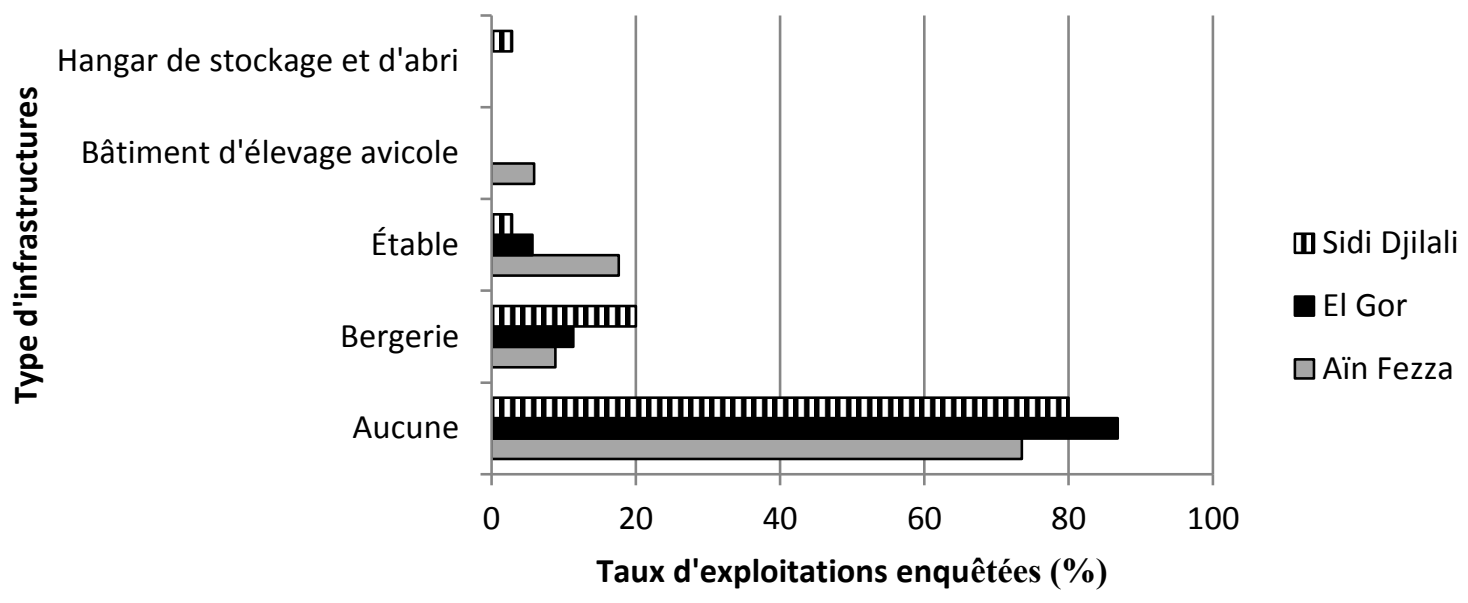

Figure 11. Taux d'exploitations agricoles enquêtées dotant d'infrastructures agricoles.

\section{L'utilisation des intrants (engrais, produits phytosanitaires et fumier)}

On remarque, selon la Figure 12, que la plupart des exploitations agricoles enquêtées à Aïn Fezza donne beaucoup d'importance à l'utilisation des intrants par rapport à El Gor et à Sidi Djilali. En pratiquant des cultures maraîchères et de l'arboriculture fruitière, les agriculteurs à Aïn Fezza se sont trouvés obligés de disposer des intrants agricoles leur permettant ainsi de bien mener ces cultures exigeantes. Quant à l'utilisation du fumier, certaines exploitations agricoles dans les régions d'El Gor et de Sidi Djilali ne donnent pas une grande importance à cette pratique qui peut améliorer significativement la productivité de leurs terres (Fig. 12). Les propriétés très utiles du fumier en matière de l'amélioration de la structure du sol, l'approvisionnement des cultures en éléments nutritifs, et surtout l'amélioration de la capacité des sols à la rétention en eau sont incontestables (Soltner, 1999). Toutefois, comme on a souligné précédemment, certains agriculteurs semblent inconscients de l'utilité du fumier notamment lorsqu'on sait que les sols en ces régions à climat aride sont peu fertiles et pauvres en matières organiques. 


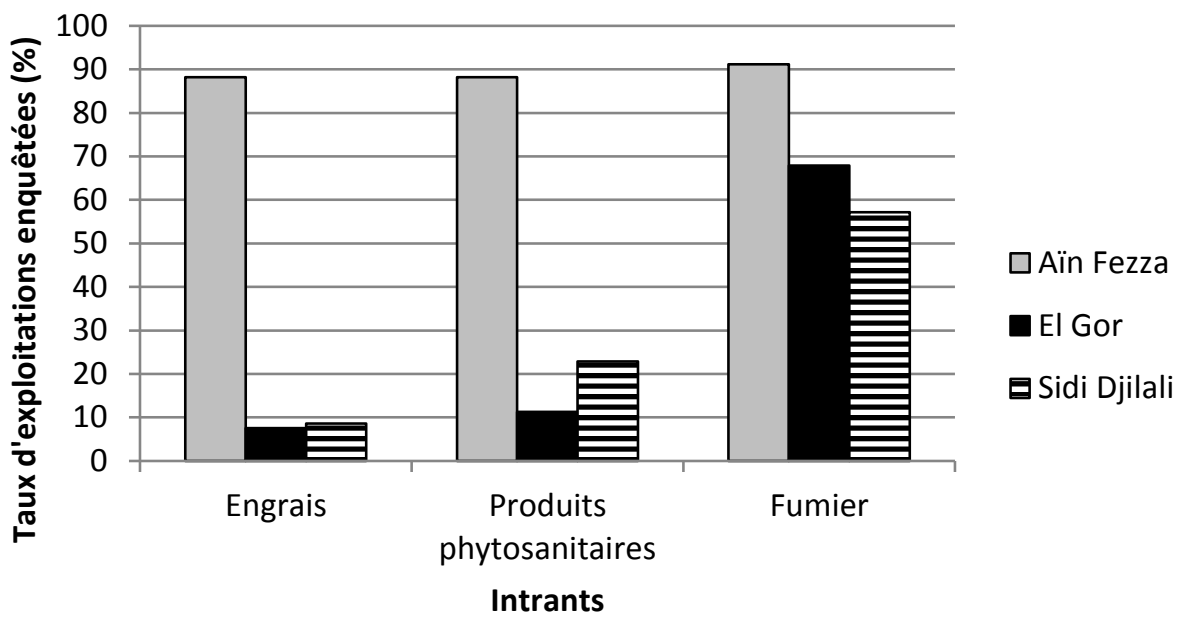

Figure 12. Taux d'exploitations agricoles enquêtées utilisant des intrants agricoles.

\section{La disponibilité en main d'œuvre salariée}

La disponibilité en main d'œuvre salariée à Aïn Fezza semble insuffisante par rapport à El Gor et à Sidi Djilali où elle paraît moyennement le contraire (Fig. 13). Le nombre moyen de main d'œuvre salariée oscille entre 07 et 10 ouvriers par exploitation agricole pour les trois régions d'étude qui sont presque tous des saisonniers (Fig. 14 et $15)$.

La rareté et la cherté de la main d'œuvre salariée à Aïn Fezza est devenue un problème qui est en train de prendre une situation alarmante ces dernières années face auquel les agriculteurs trouvent beaucoup de difficultés pour gérer leurs exploitations dans des bonnes conditions.

Dans les régions d'El Gor et de Sidi Djilali, le fait que l'agriculture est basée essentiellement sur des céréales et des fourrages conduits à l'extensif, les exploitations agricoles n'ont besoin donc de main d'œuvre salariée que pendant deux périodes: période de préparation du sol-semailles, et période de moisson-battage. C'est ce qui explique que la majorité des exploitations enquêtées ont déclaré une disponibilité suffisante en ouvriers. Or, le problème de la rareté de la main d'œuvre salariée est partout observé même en zones de plaines où les exploitations agricoles l'ont besoin durant toute la saison culturale. L'agriculture semble devenir donc un secteur dévalorisé et peu attractif à l'emploi de jeunes qui sont orientés certainement vers d'autres secteurs plus concurrents. 
Cette situation nous ramène à conclure que les exploitations agricoles notamment à Aïn Fezza sont orientées vers un autre model d'exploitation qui est l'exploitation familiale dans laquelle le travail des différentes opérations culturales sont exécutées principalement par les membres de la famille de l'exploitant. Cette tendance des exploitations agricoles montagnardes vers l'exploitation familiale a été signalée par plusieurs auteurs (Hammami et Bechir Sai, 2002 ; Revel et al., 2002 ; Bousquet, 2004 ; Wymann Von Dach et al., 2013).

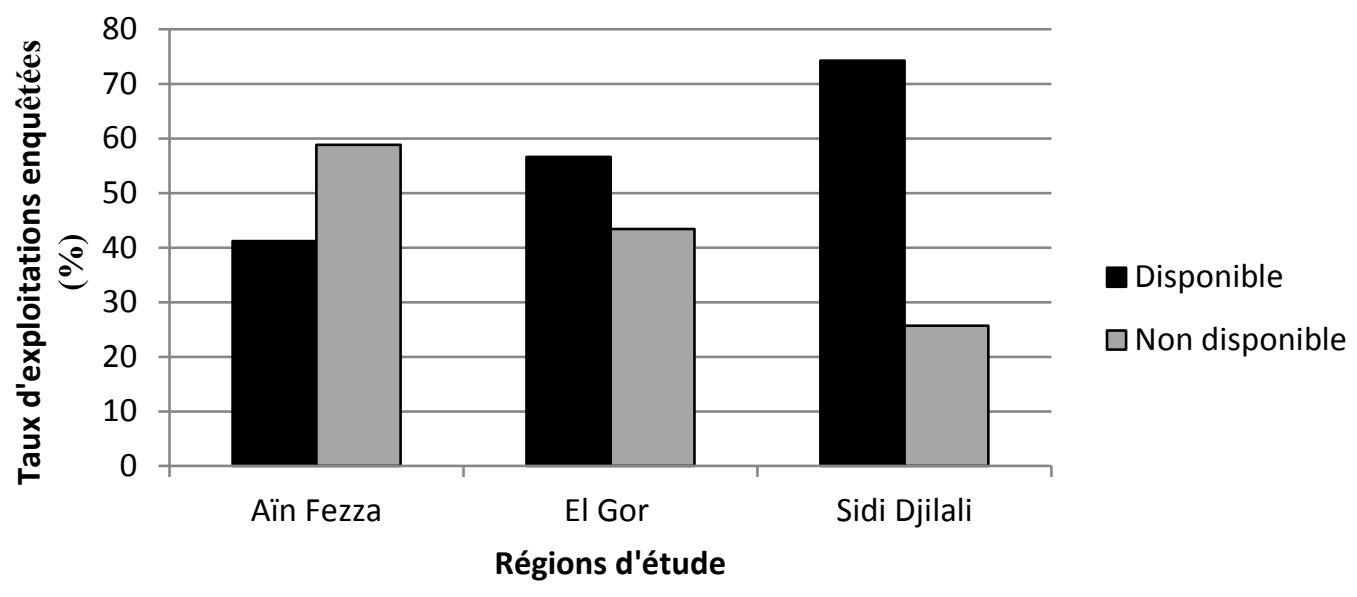

Figure 13. Taux d'exploitations agricoles enquêtées selon la disponibilité en main d'œuvre salariée.

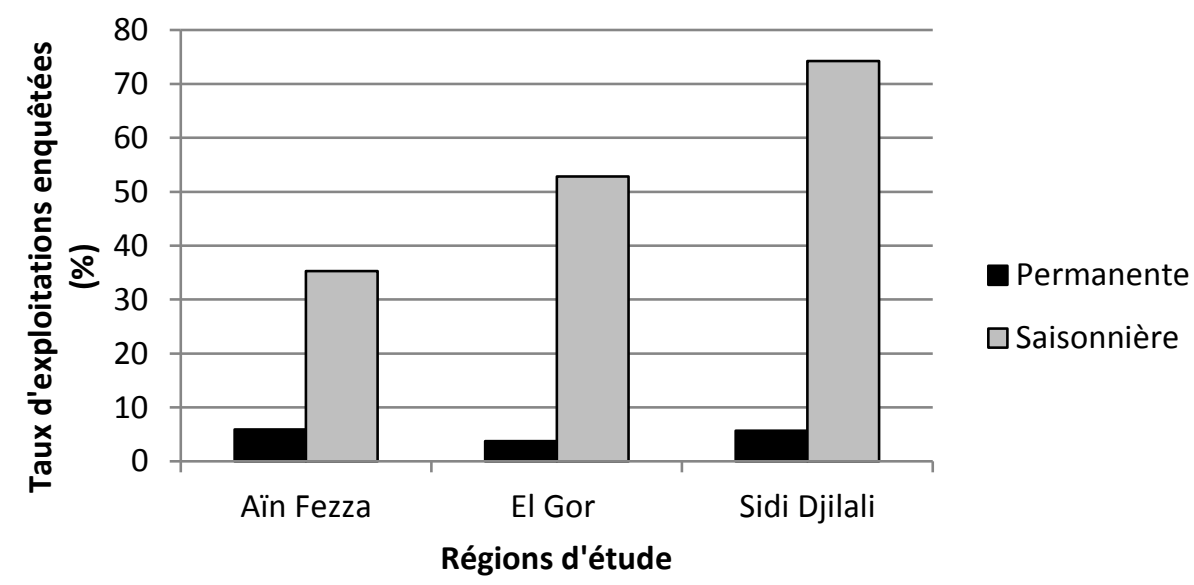

Figure 14. Taux d'exploitations agricoles enquêtées selon la nature de la main d'œuvre salariée. 


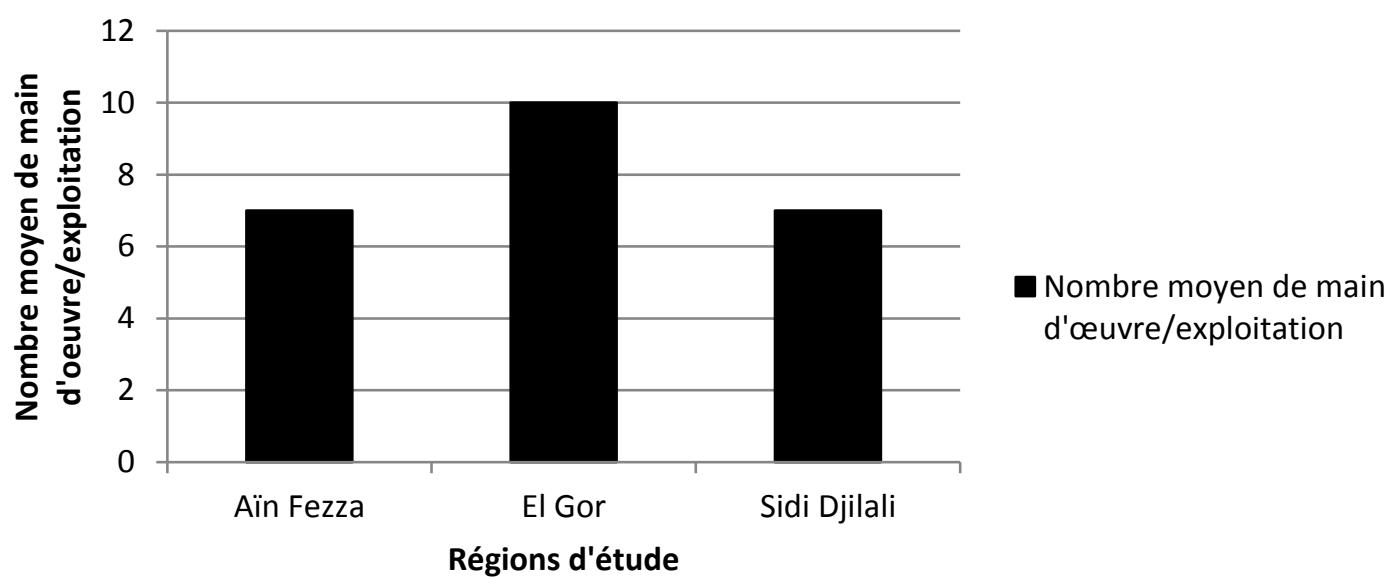

Figure 15. Nombre moyen de main d'œuvre salariée par exploitation agricole enquêtée.

\section{CONCLUSION}

Dans l'ensemble, le potentiel productif des exploitations agricoles des trois régions d'étude (équipements, infrastructures, intrants, disponibilité en main d'œuvre salariée) est insuffisant pour qu'elles puissent stabiliser la production de leurs cultures face à un climat irrégulier.

En effet, pour s'adapter aux conditions du milieu défavorables, aux moyens de production insuffisants, et surtout aux aléas climatiques et aux risques du marché fluctuant, différentes solutions peuvent être envisagées par les petits agriculteurs dans ces zones montagneuses défavorisées. Par exemple, la recherche de nouveaux points d'eau est une opération fondamentale à la création des périmètres irrigués en mobilisant des eaux souterraines (forages) et en captant et stockant des eaux superficielles (résurgences, retenues collinaires). La mise en valeur, par des procédés d'aménagement délicats, des terres accidentées à vocation agricole restant encore marginalisées, permet, de son côté, la mise en culture de ces endroits escarpés et en même temps de les sauvegarder des facteurs naturels dégradants. De même, le développement et la promotion d'autres activités agricoles notamment l'élevage de toutes sortes confondues (ovin, bovin, caprin, avicole, apicole, etc.) en parallèle avec la diversification des cultures, constituent une idéale stratégie de survie en créant ainsi des sources de revenu variées et complémentaires. L'amélioration des conditions d'approvisionnement en intrants (engrais, produits phytosanitaires, fumiers, etc.) contribue également, d'une manière significative, à l'augmentation de la productivité de ces terres agricoles. 
Finalement, les solutions susmentionnées ne seront concrétisées sur le terrain que par la participation des pouvoirs étatiques en formulant de projets de développement agricole durable spécifiques à ces zones montagneuses. Les régions d'Aïn Fezza, d'El Gor et de Sidi Djilali prises comme des échantillons à notre étude, quoiqu'elles appartiennent à un même massif montagneux, elles se distinguent l'une par rapport à l'autre par un contraste bien visible (exposition des versants, bioclimat, types de sols, nature juridique des exploitations agricoles, systèmes de production pratiqués, etc.), ce qui implique que les projets de développement agricole devraient être étudiés en zones homogènes selon les particularités communes des zones montagneuses, et que la population locale, en tant que premier acteur économique, devrait être intégrée, sous une approche participative, dans la conception, l'exécution et la gestion de ces projets.

\section{RÉFÉRENCES}

Angeliaume, A. 2011. L'eau : atout (et contrainte) pour l'agriculture de montagne. In : Antoine, J.M., Milian, J. eds. La ressource montagne entre potentialités et contraintes. Paris (France): L'HARMATTAN.

Barnaud, C., Trébuil, G., Dufumier, M. and Suphanchaimart, N. 2006. Rural Poverty and Diversification of Farming Systems in Upper Northeast Thailand. Moussons. 157-187.

Belal, E.A., Abdallah, F.E., Qishuo, D., Abaker, M. and Talha, Z. 2015. Role of animal traction in agricultural development in Zalingei area, Darfur-Sudan. Veterinaria 3: 22-27.

Benmoussa, B. 2013. An effect of globalisation? The individual appropriation of « arch » lands in Algeria. The Journal of North African Studies 5 : 668-677.

Bousquet, J.-C. 2004. La nécessaire adaptation de l'emploi agricole aux besoins de l'agriculture régionale. Montpellier Cedex: Conseil Economique et Social Languedoc-Roussillon.

Castelein, A., Dinh, T.T.V., Mekouar, M.-A. et Villeneuve, A. 2006. Les montagnes et le droit: Tendances émergentes. Rome (Italie): FAO.

FAO, 2004. L'eau, l'agriculture et l'alimentation. Rome (Italie).

FAO, 2013. Pourquoi investir dans le développement durable des montagnes ? Rome (Italie).

FAO, CIHEAM, 2007. Projet pour une agriculture et un développement rural durables en régions de montagne (ADRD-M). Rome (Italie); Bari (Italie).

Hammami, M. et Bechir Sai, M. 2002. Formation des revenus et stratégies paysannes dans les zones de montagne: le cas de Lansarine au Nord de la Tunisie. New Medit 3: 33-39.

Huang, Q., Rozelle, S., Lohmar, B., Huang, J. and Wang, J. 2006. Irrigation, agricultural performance and poverty reduction in China. Food Policy 3: 30-52. 
Jouve, A.-M. 2001. Terres méditerranéennes : le morcellement, richesse ou danger ?: Karthala-Ciheam.

Latruffe, L. and Piet, L. 2014. Does land fragmentation affect farm performance? A case study from Brittany, France. Agricultural Systems 129: 68-80.

Lawrence, P.R., Dijkman, J.T. and Jansen, H.G.P. 1997. The introduction of animal traction into inland valley regions. Manual labour and animal traction in the cultivation of rice and maize: a comparison. Journal of Agricultural Science 129: 65-70.

Lhoste, P.-h., Havard, M. et Vall, E. 2010. La traction animale. Claire Parmentier, PAG. France : CTA ; Quæ ; Presses agronomiques de Gembloux.

Lin, B.B. 2011. Resilience in agriculture through crop diversification: Adaptive management for environmental change. BioScience 61(3): 183-193.

Loi $\mathrm{n}^{\circ}$ 04-03 relative à la protection des zones de montagnes dans le cadre du développement durable. JOURNAL OFFICIEL DE LA REPUBLIQUE ALGERIENNE nº 41, 27 juin 2004.

MADR. Ministère de l'agriculture et du développement rural. 2006. Le Renouveau Rural. Alger (Algérie). $211 \mathrm{p}$.

Mihailović, B., Cvijanović, D., Milojević, I. and Filipović, M. 2014. The role of irrigation in development of agriculture in srem district. Economics of Agriculture 4: 989-1004.

Niroula, G. S. and Thapa, G. B. 2005. Impacts and causes of land fragmentation, and lessons learned from land consolidation in South Asia. Land Use Policy 22: 358372.

Revel, A., Roux, B., Bonnafous, P., Ly, B. T. et Fiack, E. 2002. Contribution au développement durable des systèmes diversifiés dans les exploitations agricoles du Languedoc-Roussillon. In : Eurocongrès des espaces occitans et catalans, (eds). Développement local, développement régional, développement durable : quelles approches? Toulouse (France) : IDEAS.

Sahli, Z. 2001. L'absence de maîtrise foncière en Algérie : impact sur l'aménagement du territoire. In : Jouve, A.M. (eds). Terres méditerranéennes : le morcellement, richesse ou danger? : Karthala-Ciheam.

Selmi, S., Araissi, N. et Zaibet, L. 2005. Irrigation et développement local Cas du périmètre irrigué du Garaat Enneam (Gouvernorat de Kasserine,Tunisie). In : Bachta, M.-S. (eds). Les instruments économiques et la modernisation des périmètres irrigués. Kairouan (Tunisia) : CIRAD.

Simon, A. 1997. La pluriactivité en agriculture, l'exemple de la moyenne Combraille. In : Cinquième colloque franco-polonais, (eds). Gestion des espaces fragiles en moyenne montagne, massif central - Carpates polonaises. Clermont-Ferrand (France) : CERAMAC. 
Simon, A. 2002. La pluriactivité dans l'agriculture des montagnes françaises: un territoire, des hommes, une pratique. Presses universitaires Blaise Pascal; Fondation Varenne. Clermont-Ferrand (France) : CERAMAC.

Soltner, D. 1999. Les bases de la production végétale. Tome 1 : Le sol et son amélioration. Collection Sciences et Techniques Agricoles. Angers (France).

Todorova, S. A. and Lulcheva, D. 2005. Economic and social effects of land fragmentation on bulgarian agriculture. Journal of Central European Agriculture 6: 555-562.

Wymann Von Dach, S., Romeo, R., Vita, A., Wurzinger, M. and Kohler, T. 2013. L'agriculture de montagne est une agriculture familiale - Une contribution des régions de montagne à l'Année internationale de l'agriculture familiale 2014 . Rome (Italie) : FAO. 